\title{
Quasi-Discrete Dynamics of a Neural Net: The Lighthouse Model
}

\author{
HERMANN HAKEN \\ Institute for Theoretical Physics 1, Center of Synergetics, Pfaffenwaldring 57/4, D-70550 Stuttgart, Germany
}

(Received 30 March 1999)

\begin{abstract}
This paper studies the features of a net of pulse-coupled model neurons, taking into account the dynamics of dendrites and axons. The axonal pulses are modelled by $\delta$-functions. In the case of small damping of dendritic currents, the model can be treated exactly and explicitly. Because of the $\delta$-functions, the phase-equations can be converted into algebraic equations at discrete times. We first exemplify our procedure by two neurons, and then present the results for $N$ neurons. We admit a general dependence of input and coupling strengths on the neuronal indices. In detail, the results are
\end{abstract}

(1) exact solution of the phase-locked state;

(2) stability of phase-locked state with respect to perturbations, such as phase jumps and random fluctuations, the correlation functions of the phases are calculated;

(3) phase shifts due to spontaneous opening of vesicles or due to failure of opening;

(4) effect of different sensory inputs on axonal pulse frequencies of coupled neurons.

Keywords: Neural nets, Discrete dynamics, Phase-locking, Fluctuations

\section{THE MODEL}

In my paper I describe a model that I recently developed [1]. It adopts a middle position between two well-known extreme models. The one widelyknown model is that of McCulloch and Pitts [2] which assumes that the neurons have only two states, one resting state and one firing state. The firing state is reached when the sum of the inputs from other neurons exceeds a certain level. The other case is represented by modelling neurons by means of the Hodgkin-Huxley model. This model, originally devised to understand the properties of axonal pulses, has been applied to the generation of pulse trains by neurons [3]. Further related models are based on the concept of integrate and fire neurons $[4,5]$. While these models deal with phase couplings via pulses, another phase coupling is achieved by the Kuramoto-type [6,7]. For recent work cf. Tass and Haken [8,9].

We first consider the generation of dendritic currents by means of axonal pulses via the synapses. 
We formulate the corresponding equation for the dendritic current $\psi$ as follows:

$$
\dot{\psi}(t)=a P(t-\tau)-\gamma \psi(t)+F_{\psi}(t),
$$

where $P$ is the axonal pulse, $\tau$ a time delay. $\gamma$ is a decay constant and $F_{\psi}$ is a fluctuating force. As is known from statistical physics, whenever there occurs damping, fluctuating forces are present. As usual we shall assume that the fluctuating forces are $\delta$-correlated in time. As is known, vesicles that release neurotransmitters and thus eventually give rise to the dendritic current can spontaneously open. This will be the main reason for the fluctuating force $F_{\psi}$. But also other noise sources may be considered here. When a pulse comes in, the opening of a vesicle occurs with only some probability. Thus we have to admit that in a more appropriate description $a$ is a randomly fluctuating quantity. While $F_{\psi}$ in (1) represents additive noise, $a$ represents multiplicative noise. In order to describe the pulses properly, we introduce a phase angle $\phi$ and connect $P$ with $\phi$ through a function $f$

$$
P(t)=f(\phi(t)) .
$$

We require the following properties of $f$ :

(a) $f(0)=0$,

(b) $f\left(\phi+\phi_{0}\right)=f(\phi)$, periodic,

(c) sharply peaked.

Finally we have to establish a relationship between the phase angle $\phi$ of the pulse $P$ produced by the neuron under consideration and the dendritic currents. To this end, we write

$$
\dot{\phi}(t)=S(X)+F_{\phi}(t),
$$

where the function $S(X)$ has the following properties: $S$ is equal to zero for $X$ smaller than a threshold $\Theta$, then it increases in a quasi-linear fashion until it saturates. Denoting the dendritic currents of other neurons by $\psi_{m}$, we write $S$ in the form

$$
S(X)=S\left(\sum_{m} c_{m} \psi_{m}\left(t-\tau^{\prime}\right)+p_{\mathrm{ext}}\left(t-\tau^{\prime \prime}\right)-\Theta\right) .
$$

Here, $\tau^{\prime}$ and $\tau^{\prime \prime}$ are delay times. $p_{\text {ext }}$ is an external signal that is transferred to the neuron under consideration from sensory neurons. A simple explicit representation of (7) obeying the properties just required for $S$ is given by

$$
\begin{aligned}
& \dot{\phi}=\sum_{m} c_{m} \psi_{m}\left(t-\tau^{\prime}\right)+p_{\text {ext }}\left(t-\tau^{\prime \prime}\right)-\Theta \text { for } S>0, \\
& \dot{\phi}=0 \\
& \phi=0\} \\
& \text { otherwise. }
\end{aligned}
$$

The interpretation of Eq. (6) is based on the functioning of a lighthouse, in which a light beam rotates. The rotation speed $\dot{\phi}$ depends on $S$ according to (6). The fluctuating forces $F_{\phi}$ lead to a shift of the phase at random instances. The relationships (1), (2) and (7) can be easily generalized to the equations of a whole network. The index $m$ or $k$ refers to the location and to the property "excitatory" or "inhibitory". The generalizations are straightforward and read

$$
\begin{gathered}
\dot{\psi}_{m}(t)=\sum_{k} a_{m k} P_{k}(t-\tau)-\gamma \psi_{m}(t)+F_{\psi, m}(t), \\
P_{k}(t)=f\left(\phi_{k}(t)\right) \\
\dot{\phi}_{k}(t)=S\left(\sum_{m} c_{k m} \psi_{m}\left(t-\tau^{\prime}\right)\right. \\
\left.+p_{\mathrm{ext}, k}\left(t-\tau^{\prime \prime}\right)-\Theta_{k}\right)+F_{\phi, k}(t) .
\end{gathered}
$$

\section{TWO NEURONS: BASIC EQUATIONS FOR THE IMPACT OF PERTURBATIONS}

We first make the conditions (3)-(5) of Section 1 more explicit by using the representation

$$
f(\phi)=\sum_{n=-\infty}^{+\infty} g(\phi, \dot{\phi}) \delta\left(\phi-\phi_{n}\right),
$$


whereby

$$
\phi_{n}=2 \pi n-\delta, \quad 0<\delta<2 \pi, \quad \phi\left(t_{n}\right)=\phi_{n} .
$$

We choose the function $g$ such that

$$
\int_{t_{n}-\epsilon}^{t_{n}+\epsilon} g \delta\left(\phi(t)-\phi_{n}\right) \mathrm{d} t=1 \quad \text { for } t \in\left(t_{n}-\epsilon, t_{n}+\epsilon\right)
$$

and

$$
=0 \text { for } t \notin\left(t_{n}, t_{n+1}\right) \text {. }
$$

An explicit form of $g$ reads

$$
g=\dot{\phi}(t)
$$

because

$$
\int_{t_{n}-\epsilon}^{t_{n}+\epsilon} \dot{\phi} \delta\left(\phi(t)-\phi_{n}\right) \mathrm{d} t=\int_{\phi_{n}-\delta}^{\phi_{n}+\delta} \delta\left(\phi-\phi_{n}\right) \mathrm{d} \phi
$$

where $\phi_{n} \pm \delta=\phi\left(t_{n} \pm \epsilon\right)$.

We start from (9)-(11) and assume that the system operates in the linear regime of $S$ (cf. (8)). In this section we neglect delays, i.e. we put $\tau=\tau^{\prime}=0$. By differentiating (11) with respect to time, we may eliminate $\psi_{1}, \dot{\psi}_{1}$ from (9) and (11), thus obtaining

$$
\ddot{\phi}_{1}+\gamma \dot{\phi}_{1}=A f\left(\phi_{2}\right)+C_{1},
$$

for neuron 1, where

$$
C_{1}=\gamma\left(p_{\mathrm{ext}, 1}-\Theta\right)+\dot{p}_{\mathrm{ext}, 1}
$$

Similarly, we obtain for neuron 2 :

$$
\ddot{\phi}_{2}+\gamma \dot{\phi}_{2}=A f\left(\phi_{1}\right)+C_{2},
$$

where

$$
C_{2}=\gamma\left(p_{\mathrm{ext}, 2}-\Theta\right)+\dot{p}_{\mathrm{ext}, 2}
$$

We integrate (18) and (19) over time $t$ and observe in (9)-(11) the initial conditions $\phi(0)=0, \psi(0)=0$ jointly with $f(0)=0$ (cf. (3))

$$
\begin{aligned}
& \dot{\phi}_{1}(t)-\dot{\phi}_{1}(0)+\gamma \phi_{1}(t) \\
& =A \int_{0}^{t} f\left(\phi_{2}\right) \mathrm{d} t+\int_{0}^{t} C_{1}(t) \mathrm{d} t
\end{aligned}
$$

where $\dot{\phi}_{1}(0)=p_{\text {ext }, 1}(0)-\Theta$. Using $(21)$, we rearrange this equation:

$$
\begin{aligned}
\dot{\phi}_{1}(t) & +\gamma \phi_{1}(t) \\
= & A \int_{0}^{t} f\left(\phi_{2}\right) \mathrm{d} t+\int_{0}^{t} \gamma\left(p_{\mathrm{ext}, 1}-\Theta\right) \mathrm{d} t \\
& +p_{\mathrm{ext}, 1}(t)-\Theta .
\end{aligned}
$$

For what follows we put the last three terms on the r.h.s. of (23)

$$
=C t+B_{1}(t)
$$

We proceed with (20) in complete analogy. We now evaluate

$$
\begin{aligned}
\int_{0}^{t} f(\phi) \mathrm{d} t & \equiv \int_{0}^{t} \dot{\phi} \sum_{n} \delta\left(\phi-\phi_{n}\right) \mathrm{d} t \\
& =\sum_{n} \int^{\phi} \delta\left(\phi-\phi_{n}\right) \mathrm{d} \phi
\end{aligned}
$$

and obtain

$$
(25)=\sum_{n} H\left(\phi-\phi_{n}\right)
$$

where

$$
\begin{aligned}
H(\sigma) & =0 & & \text { for } \sigma<0 \\
& =\frac{1}{2} & & \text { for } \sigma=0 \\
& =1 & & \text { for } \sigma>0
\end{aligned}
$$

Equation (26) represents a series of step functions each of height 1 . An equivalent representation of (26) and (27), leaving out the points with $\sigma=0$ is

$$
2 \pi \cdot(26)=\phi-\phi \bmod 2 \pi
$$

where it is understood that an integer $n$ is chosen such that

$$
\phi \bmod 2 \pi=\phi-2 \pi n
$$


so that

$$
0 \leq \phi-2 \pi n<2 \pi .
$$

Lumping all steps following (22) together, and doing the same with the equation for $\phi_{2}$, we obtain

$$
\begin{aligned}
& \dot{\phi}_{1}+\gamma \phi_{1}=\tilde{A}\left(\phi_{2}-\phi_{2} \bmod 2 \pi\right)+C t+B_{1}(t), \\
& \dot{\phi}_{2}+\gamma \phi_{2}=\tilde{A}\left(\phi_{1}-\phi_{1} \bmod 2 \pi\right)+C t+B_{2}(t),
\end{aligned}
$$

where $\tilde{A}=A /(2 \pi)$. Since we are particularly interested in phase-locking, i.e. $\phi_{2}=\phi_{1}$, we introduce the corresponding equation

$$
\dot{\phi}+\gamma \phi=\tilde{A}(\phi-\phi \bmod 2 \pi)+C t .
$$

We further put

$$
\phi_{j}=\phi+\xi_{j}, \quad j=1,2 .
$$

Subtracting (33) from (32), we obtain

$$
\begin{aligned}
\dot{\xi}_{2}+\gamma \xi_{2}= & -\tilde{A}\left[\phi-\phi \bmod 2 \pi-\left(\left(\phi+\xi_{1}\right)\right.\right. \\
& \left.\left.-\left(\phi+\xi_{1}\right) \bmod 2 \pi\right)\right]+B_{2}
\end{aligned}
$$

A corresponding equation results for $\xi_{1}$ and each of the following transformations must be performed also with that equation. We abbreviate the square bracket in (35) by $k\left(\phi, \xi_{1}\right)$ and integrate (35) over time observing $\xi_{2}(0)=0$

$$
\begin{aligned}
\xi_{2}(t)= & \int_{0}^{t} \mathrm{e}^{-\gamma(t-\sigma)} B_{2}(\sigma) \mathrm{d} \sigma \\
& -\tilde{A} \underbrace{\int_{0}^{t} \mathrm{e}^{-\gamma(t-\sigma)} k\left(\phi, \xi_{1}\right)_{\sigma} \mathrm{d} \sigma}_{\kappa(t)} .
\end{aligned}
$$

In this section we shall assume that $B_{j}$ is bounded and small enough so that

$$
\left|\xi_{j}\right|<\pi, \quad j=1,2 .
$$

We first assume that

$$
\xi_{1}>0
$$

holds. We study the properties of $k$ in (36) and first assume $\xi_{1} \geq 0$ :

(1) be $2 \pi n<\phi<2 \pi(n+1)$,

$$
2 \pi n<\phi+\xi_{1}<2 \pi(n+1),
$$

where $n$ are integers,

then

$$
k=0
$$

(2) (because of $\xi_{1}>0$ )

$$
\begin{gathered}
2 \pi n<\phi<2 \pi(n+1), \\
2 \pi(n+1)<\phi+\xi_{1}<2 \pi(n+2), \\
k=\phi-(\phi-2 \pi n)-\left(\left(\phi+\xi_{1}\right)\right. \\
\left.-\left(\phi+\xi_{1}\right)+2 \pi(n+1)\right)=-2 \pi
\end{gathered}
$$

(3)

$$
\xi_{1}=0,
$$

$k=0$ independent of interval $n$.

We assume that $\phi(t)$ increases monotonously. We study the behavior of $\kappa(t)$ in (36), beginning with $t=0$ :

(1)

$$
t_{0}=0, \quad \xi_{1}(0)=0
$$

for

$$
t>0, \quad \xi_{1}(t)>0
$$

and conditions (39) and (40) are fulfilled till a time $t_{1}^{-}$. Till then $k=0$,

(2)

$$
\text { for } t_{1}^{-} \leq t, \quad \xi_{1}(t)>0, \quad k=-2 \pi, \quad \text { and }
$$

$$
\begin{aligned}
\kappa(t) & =-2 \pi \int_{t_{1}^{-}}^{t} \mathrm{e}^{-\gamma(t-\sigma)} \mathrm{d} \sigma \\
& =-\frac{2 \pi}{\gamma}\left(1-\mathrm{e}^{-\gamma\left(t-t_{1}^{-}\right)}\right) \mathrm{d} \sigma ;
\end{aligned}
$$


(3)

$$
\text { for } t_{1}^{+} \leq t
$$

(a) either $\xi_{1}\left(t_{1}^{+}\right)=0(($ condition 3$)(44))$, and same procedure as before, or

(b) $\xi_{1}\left(t_{1}^{+}\right)>0$ and condition 1(39) fulfilled so that $k=0$,

$$
\kappa(t)=-\frac{2 \pi}{\gamma} \mathrm{e}^{-\gamma t}\left(\mathrm{e}^{\gamma t_{1}^{+}}-\mathrm{e}^{\gamma t_{1}^{-}}\right)
$$

we may proceed in analogy to 2 and obtain

(4)

$$
\begin{aligned}
t_{2}^{-} \leq t & \\
\kappa(t) & =-2 \pi \int_{t_{1}^{-}}^{t_{1}^{+}} \mathrm{e}^{-\gamma(t-\sigma)} \mathrm{d} \sigma-2 \pi \int_{t_{2}^{-}}^{t} \mathrm{e}^{-\gamma(t-\sigma)} \mathrm{d} \sigma \\
& =-\frac{2 \pi}{\gamma}\left\{\mathrm{e}^{-\gamma t}\left(\mathrm{e}^{\gamma t_{1}^{+}}-\mathrm{e}^{\gamma t_{1}^{-}}\right)+1-\mathrm{e}^{-\gamma\left(t-t_{2}^{-}\right)}\right\}
\end{aligned}
$$

For $t_{L+1}^{-}<t<t_{L+1}^{+}$, the general result reads

$$
\begin{aligned}
\kappa(t)= & -\frac{2 \pi}{\gamma}\left(\mathrm{e}^{-\gamma t} \sum_{\ell=1}^{L}\left(\mathrm{e}^{\gamma t_{\ell}^{+}}-\mathrm{e}^{\gamma t_{\ell}^{-}}\right)\right. \\
& \left.+1-\mathrm{e}^{-\gamma\left(t-t_{L+1}^{-}\right)}\right) .
\end{aligned}
$$

For later purposes, we quote an alternative representation of $\kappa(t)$, namely

$$
\begin{aligned}
\kappa(t)= & -\frac{2 \pi}{\gamma} \int_{0}^{t} \mathrm{e}^{-\gamma(t-\sigma)} \sum_{\ell=-\infty}^{+\infty} \delta\left(\sigma-t_{\ell}^{+}\right) \\
& \times\left(1-\mathrm{e}^{\gamma\left(t_{\ell}^{-}-t_{\ell}^{+}\right)}\right) \mathrm{d} \sigma .
\end{aligned}
$$

Let us discuss the times $t_{n}^{-}$and $t_{n}^{+}$in more detail. These times are defined by

$$
\begin{aligned}
& t_{n}^{-}: \phi\left(t_{n}^{-}\right)+\xi_{1}\left(t_{n}^{-}\right)=2 \pi n, \\
& t_{n}^{+}: \phi\left(t_{n}^{+}\right)=2 \pi n .
\end{aligned}
$$

Subtracting (51) from (50) yields $\phi\left(t_{n}^{-}\right)-\phi\left(t_{n}^{+}\right)+$ $\xi_{1}\left(t_{n}^{-}\right)=0$. If $\xi_{1}$ is small (and $\phi$ differentiable), we may assume

$$
\phi\left(t_{n}^{-}\right)-\phi\left(t_{n}^{+}\right) \approx \dot{\phi}\left(t_{n}^{-}\right)\left(t_{n}^{-}-t_{n}^{+}\right)
$$

and further

$$
\dot{\phi}\left(t_{n}^{-}\right)\left(t_{n}^{-}-t_{n}^{+}\right)+\xi_{1}\left(t_{n}^{-}\right)=0 .
$$

Thus we obtain

$$
\left(t_{n}^{-}-t_{n}^{+}\right)=-\dot{\phi}\left(t_{n}^{-}\right)^{-1} \xi_{1}\left(t_{n}^{-}\right) .
$$

To the same degree of approximation we obtain

$$
\left(t_{n}^{-}-t_{n}^{+}\right)=-\dot{\phi}\left(t_{n}^{+}\right)^{-1} \xi_{1}\left(t_{n}^{-}\right) .
$$

A closer inspection of our above procedure shows that this holds both for $t_{n}^{-}<t_{n}^{+}$and $t_{n}^{-}>t_{n}^{+}$, i.e. for both positive and negative $\xi_{1}$.

We are now in a position to discuss the effect of (49) or (48) in our basic equation for $\xi_{1}(t)(36)$. We note that $\mathrm{e}^{-\gamma(t-\sigma)}$ is the Green's function of the equation

$$
\dot{\xi}+\gamma \xi=F(t),
$$

i.e. $\xi(t)=\int_{0}^{t} \mathrm{e}^{-\gamma(t-\sigma)} F(\sigma) \mathrm{d} \sigma$.

This allows us to transform (36) with (49) into the equation

$\dot{\xi}_{2}+\gamma \xi_{2}$

$$
=B_{2}(t)+\frac{A}{\gamma} \sum_{\ell=-\infty}^{\infty} \delta\left(t-t_{\ell}^{+}\right)\left(1-\mathrm{e}^{\gamma\left(t_{\ell}^{-}-t_{\ell}^{+}\right)}\right) .
$$

Using (54) and the property of the $\delta$-function, we obtain

$$
\begin{aligned}
\dot{\xi}_{2}+\gamma \xi_{2}= & B\left(t_{2}\right)+\frac{A}{\gamma} \sum_{\ell=-\infty}^{\infty} \delta\left(t-t_{\ell}^{+}\right) \\
& \times\left\{1-\exp \left(-\gamma \dot{\phi}(t)^{-1} \xi_{1}(t)\right)\right\},
\end{aligned}
$$

where we made the approximation $\xi_{1}\left(t_{l}^{-}\right) \approx$ $\xi_{1}\left(t_{l}^{+}\right)=\xi_{1}(t)$. 
Because of its r.h.s., (56) and the equation with indices 1 and 2 exchanged are highly nonlinear equations for $\xi_{j}(t)$ that can be solved only numerically. If $\left|\gamma \dot{\phi}(t)^{-1} \xi_{1}\right|$ is small, however, (56) and its corresponding equation acquire a very simple form, namely

$$
\begin{aligned}
& \dot{\xi}_{2}+\gamma \xi_{2}=a D(t) \xi_{1}+B_{2}(t), \\
& \dot{\xi}_{1}+\gamma \xi_{1}=a D(t) \xi_{2}+B_{1}(t),
\end{aligned}
$$

where $a=A \dot{\phi}^{-1}$ and $D(t)=\sum_{l} \delta\left(t-t_{l}^{+}\right)$, which is a known function, where $t_{l}^{+}$is defined by $\phi\left(t_{l}^{+}\right)=$ $2 \pi l$. Adding or subtracting Eq. (57) from each other, we obtain

$$
\dot{\sum}+\gamma \sum=a D(t) \sum+B_{+}
$$

where

$$
\sum=\xi_{1}+\xi_{2}, \quad B_{+}=B_{1}+B_{2}
$$

and

$$
\dot{\xi}+\gamma \xi=-a D(t) \xi+B,
$$

where $\xi=\xi_{2}-\xi_{1}, B=B_{2}-B_{1}$, respectively.

\section{PHASE RELAXATION AND THE IMPACT OF NOISE}

In the preceding section we derived equations for the phase-deviation $\xi_{j}(t)$ from the phase-locked state. Equation (58) refers to the phase-difference $\xi \equiv \xi_{2}-\xi_{1} \equiv \phi_{2}-\phi_{1}$ and reads (with $B=0$ )

$$
\dot{\xi}(t)+\gamma \xi(t)=-a \sum \delta\left(t-t_{n}\right) \xi(t) .
$$

In the following we again use the abbreviation:

$$
A / \dot{\phi}=a \text {. }
$$

Because of the $\delta$-functions in (59), $\dot{\phi}$ is to be taken at the discrete times $t_{n}$. Because the phase $\phi$ refers to the steady state, $a$ in (60) is a constant. We first study the solution of (60) in the interval

$$
t_{n}<t<t_{n+1}
$$

and obtain

$$
\xi(t)=\xi\left(t_{n}+\epsilon\right) \mathrm{e}^{-\gamma\left(t-t_{n}\right)} .
$$

At times $t_{n}$ we integrate (59) over a small interval around $t_{n}$ and obtain

$$
\xi\left(t_{n}+\epsilon\right)=\xi\left(t_{n}-\epsilon\right)-a \xi\left(t_{n}-\epsilon\right) .
$$

Since $\xi$ undergoes a jump at time $t_{n}$, there is an ambiguity with respect to the evaluation of the last term in (63). Instead of $t_{n}-\epsilon$ we might equally well choose $t_{n}+\epsilon$ or an average over both expressions. Since we assume, however, that $a$ is a small quantity, the error is of higher order and we shall, therefore, choose $\xi$ at $t_{n}-\epsilon$ as shown in Eq. (63). (Taking the average amounts to replacing $(1-a)$ by $(1-a / 2) /(1+a / 2)$.) On the r.h.s. of (63), we insert (62) for $t=t_{n}+\epsilon$ and thus obtain

$$
\xi\left(t_{n}+\epsilon\right)=(1-a) \xi\left(t_{n-1}+\epsilon\right) \mathrm{e}^{-\gamma\left(t_{n}-t_{n-1}\right)} .
$$

Since the $t_{n}$ 's are equally spaced, we put

$$
t_{n}-t_{n-1} \equiv \Delta .
$$

For the interval

$$
t_{N}<t<t_{N+1}
$$

the solution reads

$$
\xi(t)=\xi\left(t_{0}+\epsilon\right)(1-a)^{N} \mathrm{e}^{-\gamma \Delta \cdot N-\gamma\left(t-t_{N}\right)} .
$$

Since the absolute value of $1-a$ is smaller than unity, (67) shows that the phase deviation $\xi(t)$ relaxes towards zero in the course of time.

We now study the impact of noise in which case Eq. (59) becomes

$$
\dot{\xi}(t)+\gamma \xi(t)=B(t)-a \sum \delta\left(t-t_{n}\right) \xi(t) .
$$


In the interval

$$
t_{n-1}<t<t_{n}
$$

the general solution of (68) reads

$\xi(t)=\xi\left(t_{n-1}+\epsilon\right) \mathrm{e}^{-\gamma\left(t-t_{n-1}\right)}+\int_{t_{n-1}}^{t} \mathrm{e}^{-\gamma(t-\sigma)} B(\sigma) \mathrm{d} \sigma$.

We first treat the case that $B(t)$ is nonsingular. At time $t_{n}$, the integration of (68) over a small time interval yields

$$
\xi\left(t_{n}+\epsilon\right)=\xi\left(t_{n}-\epsilon\right)-a \xi\left(t_{n}-\epsilon\right) .
$$

We put $t=t_{n}-\epsilon$ in (70) and thus obtain

$$
\begin{aligned}
\xi\left(t_{n}-\epsilon\right)= & \xi\left(t_{n-1}+\epsilon\right) \mathrm{e}^{-\gamma\left(t_{n}-t_{n-1}\right)} \\
& +\int_{t_{n-1}}^{t_{n}} \mathrm{e}^{-\gamma\left(t_{n}-\sigma\right)} B(\sigma) \mathrm{d} \sigma .
\end{aligned}
$$

We now replace the r.h.s. of (71) by means of (72) and obtain

$$
\xi\left(t_{n}+\epsilon\right)=(1-a)\left\{\xi\left(t_{n-1}+\epsilon\right) \mathrm{e}^{-\gamma \Delta}+\hat{B}\left(t_{n}\right)\right\},
$$

where we abbreviated the integral in (72) by $\hat{B}$. Introducing the variable $x$ instead of $\xi$, we can rewrite (73) in an obvious manner by means of

$$
x_{n}=(1-a)\left\{x_{n-1} \mathrm{e}^{-\gamma \Delta}+\hat{B}_{n}\right\} .
$$

To solve the set of Eq. (74), we make the substitution

$$
x_{n}=\left((1-a) \mathrm{e}^{-\gamma \Delta}\right)^{n} y_{n}
$$

and obtain a recursion formula for $y_{n}$,

$$
y_{n}-y_{n-1}=(1-a)^{-n+1} \mathrm{e}^{\gamma t_{n}} \hat{B}_{n} .
$$

Summing up over both sides of (76), we obtain

$$
\sum_{n=1}^{N}\left(y_{n}-y_{n-1}\right)=\sum_{n=1}^{N}(1-a)^{-n+1} \mathrm{e}^{\gamma t_{n}} \hat{B}_{n}
$$

or written more explicitly

$$
y_{N}=y_{0}+\sum_{n=1}^{N}(1-a)^{-n+1} \int_{t_{n-1}}^{t_{n}} \mathrm{e}^{\gamma \sigma} B(\sigma) \mathrm{d} \sigma .
$$

By means of (75), we obtain the final result in the form (with $t_{n}-t_{n-1}=\Delta$ )

$$
\begin{aligned}
x_{N}= & y_{0}\left((1-a) \mathrm{e}^{-\gamma \Delta}\right)^{N} \\
& +\sum_{n=1}^{N}(1-a)^{N-n+1} \mathrm{e}^{-\gamma \Delta N} \int_{t_{n-1}}^{t_{n}} \mathrm{e}^{\gamma \sigma} B(\sigma) \mathrm{d} \sigma .
\end{aligned}
$$

In order to evaluate (79), we need the stochastic properties of $B$. Before we proceed further, we discuss the case in which $B(t)$ is singular, for instance of the form

$$
B \delta\left(t-t_{n_{0}}\right) .
$$

For $t<t_{0}$ we can proceed in analogy to the Eqs. (59)-(67). For

$$
t \geq t_{n_{0}}
$$

the integration of Eq. (68) around $t=t_{n_{0}}$ yields

$$
\xi\left(t_{n_{0}}+\epsilon\right)=\xi\left(t_{n_{0}}-\epsilon\right)-a \xi\left(t_{n_{0}}-\epsilon\right)+B
$$

and in between the singularities we have as solution of (68),

$$
\xi\left(t_{n}-\epsilon\right)=\xi\left(t_{n-1}+\epsilon\right) \mathrm{e}^{-\gamma\left(t-t_{n-1}\right)} .
$$

To be still more specific let us assume that for

$$
t<t_{n_{0}}
$$

the solution reads

$$
\xi\left(t_{n}\right)=0 .
$$

Then instead of (82), we obtain

$$
\xi\left(t_{n_{0}}+\epsilon\right)=B,
$$


which means that we now proceed as we did it following Eq. (59), namely (86) acts just as initial condition.

We now treat the case in which $B$ is time independent. The integral in (79) can immediately be evaluated and we obtain

$$
\begin{aligned}
x_{N} & =y_{0}\left((1-a) \mathrm{e}^{-\gamma \Delta}\right)^{N} \\
& +\sum_{n=1}^{N}(1-a)^{N-n+1} \mathrm{e}^{-\gamma \Delta N} B \frac{1}{\gamma}\left(\mathrm{e}^{\gamma t_{n}}-\mathrm{e}^{\gamma t_{n-1}}\right) .
\end{aligned}
$$

The explicit evaluation of that sum is a simple matter and we obtain (with $x_{0}=y_{0}$ )

$$
\begin{aligned}
x_{N}= & x_{0}(1-a)^{N} \mathrm{e}^{-\gamma \Delta N}+\frac{B}{\gamma}\left(\mathrm{e}^{\gamma \Delta}-1\right) \\
& \times \frac{1-(1-a)^{N} \mathrm{e}^{-\gamma \Delta N}}{(1-a) \mathrm{e}^{-\gamma \Delta}-1} .
\end{aligned}
$$

It tells us that the effect of the perturbation persists, and that $x_{N}$ eventually acquires a constant value.

We now turn to the case in which $B$ is a stochastic function of time, where we shall assume that the statistical average over $B$ vanishes. In the following we shall study the correlation function for the case $N$ large, and

$$
\left|N-N^{\prime}\right| \text { finite. }
$$

Using (79), the correlation function can be written in the form

$$
\begin{aligned}
& \left\langle x_{N} x_{N^{\prime}}\right\rangle \\
& =\sum_{n=1}^{N} \sum_{n^{\prime}=1}^{N^{\prime}}(1-a)^{N-n+1} \mathrm{e}^{-\gamma \Delta N}(1-a)^{N^{\prime}-n^{\prime}+1} \\
& \quad \times \mathrm{e}^{-\gamma \Delta N^{\prime}} \int_{t_{n-1}}^{t_{n}} \int_{t_{n^{\prime}}-1}^{t_{n^{\prime}}} \mathrm{d} \sigma \mathrm{d} \sigma^{\prime} \\
& \quad \times \mathrm{e}^{\gamma \sigma} \mathrm{e}^{\gamma \sigma^{\prime}}\left\langle B(\sigma) B\left(\sigma^{\prime}\right)\right\rangle .
\end{aligned}
$$

We evaluate (90) in the case

$$
N^{\prime} \geq N
$$

and assume further that $B$ is $\delta$-correlated with strength $Q$. Then (90) acquires the form

$$
\begin{aligned}
R \equiv & \left\langle x_{N} x_{N^{\prime}}\right\rangle \\
= & \sum_{n=1}^{N}(1-a)^{N-n+1} \mathrm{e}^{-\gamma \Delta N}(1-a)^{N^{\prime}-n+1} \\
& \times \mathrm{e}^{-\gamma \Delta N^{\prime}} \frac{1}{2 \gamma}\left(\mathrm{e}^{2 \gamma t_{n}}-\mathrm{e}^{2 \gamma t_{n-1}}\right) Q .
\end{aligned}
$$

The evaluation of the sum in (92) is straightforward and yields

$$
\begin{aligned}
& \frac{Q}{2 \gamma}\left(\mathrm{e}^{2 \gamma \Delta}-1\right)\left\{(1-a)^{-2} \mathrm{e}^{2 \gamma \Delta}-1\right\}^{-1} \\
& \quad \times(1-a)^{N^{\prime}-N} \mathrm{e}^{-\gamma \Delta\left(N^{\prime}-N\right)},
\end{aligned}
$$

which for

$$
a \ll 1,
$$

can be written as

$$
R=\mathrm{e}^{-(\gamma \Delta+a)\left(N^{\prime}-N\right)} \cdot \frac{Q}{2 \gamma} .
$$

The correlation function has the same form as we would expect it from a purely continuous treatment of the Eq. (68), i.e. in which the $\delta$-functions are smeared out.

\section{TWO NEURONS: EXPLICIT SOLUTION OF THE PHASE-LOCKED STATE}

In the preceding section we studied a variety of deviations from the phase-locked state, of which we needed only a few general properties. In this section we wish to explicitly construct that function. Its equation is of the form

$$
\ddot{\phi}+\gamma \dot{\phi}=A f(\phi)+C .
$$

By making the transformation

$$
\phi=\frac{1}{\gamma} C t+\chi=c t+\chi,
$$


we can cast (96) into

$$
\ddot{\chi}+\gamma \dot{\chi}=A f(\chi+c t)
$$

We note that $\chi$ is continuous everywhere

$$
\chi(t+\epsilon)=\chi(t-\epsilon) .
$$

On the other hand, because of the singular character of (12), which is explicitly expressed by (14) and (15), integrating (98) over the time-interval $\left(t_{n+1}-\epsilon, t_{n+1}+\epsilon\right)$, we immediately obtain

$$
\dot{\chi}\left(t_{n+1}+\epsilon\right)-\dot{\chi}\left(t_{n+1}-\epsilon\right)=A \text { for } t=t_{n+1} \text {. }
$$

On the other hand, for the time-interval $t_{n}+\epsilon \leq$ $t \leq t_{n+1}-\epsilon$, we obtain

$$
\ddot{\chi}+\gamma \dot{\chi}=0, \quad \dot{\chi}(t)=\dot{\chi}\left(t_{n}+\epsilon\right) \cdot \mathrm{e}^{-\gamma\left(t-t_{n}\right)} .
$$

Using (101) in (100), we obtain the recursive relation

$$
\dot{\chi}\left(t_{n+1}+\epsilon\right)=\dot{\chi}\left(t_{n}+\epsilon\right) \mathrm{e}^{-\gamma\left(t_{n+1}-t_{n}\right)}+A .
$$

We first assume that the times $t_{n}$ at which the jumps of the derivatives of the phase occur are given quantities. In the following we shall study (102) explicitly. We first introduce the abbreviations

$$
x_{n}=\dot{\chi}\left(t_{n}+\epsilon\right)
$$

that allows us to cast (102) into the form

$$
x_{n+1}=x_{n} \mathrm{e}^{-\gamma\left(t_{n+1}-t_{n}\right)}+A \text {. }
$$

By using the substitution

$$
x_{n} \mathrm{e}^{\gamma t_{n}}=y_{n},
$$

we cast (104) into the form

$$
y_{n+1}-y_{n}=A \mathrm{e}^{\gamma t_{n+1}} \text {. }
$$

Summing (106) over both sides, yields

$$
\begin{aligned}
\sum_{n=0}^{N-1}\left(y_{n+1}-y_{n}\right) & =\sum_{n=0}^{N-1} A \mathrm{e}^{\gamma t_{n+1}} \\
& =\sum_{n=1}^{N} A \mathrm{e}^{\gamma t_{n}} \equiv Z_{N},
\end{aligned}
$$

or because of the cancellation of terms on the 1.h.s. of (107)

$$
y_{N}=y_{0}+Z_{N}
$$

Because of (105), (108) can be cast into the form

$$
x_{N}=\mathrm{e}^{-\gamma t_{N}} x_{0}+\sum_{n=1}^{N} A \mathrm{e}^{-\gamma\left(t_{N}-t_{n}\right)} .
$$

Note that

$$
x_{N} \equiv \dot{\chi}\left(t_{N}+\epsilon\right)
$$

Because the dependence of the jump-times on the phases $\phi$ is not specified, the solution (109) is valid quite generally. Introducing a time $T$ so that

$$
t_{N}<T<t_{N+1}
$$

holds, we may write $x(T)$ at that general time in the form

$$
x(T)=\mathrm{e}^{-\gamma\left(T-t_{N}\right)} x\left(t_{N}\right) .
$$

We now study the relationship between the jumptimes or their difference, i.e.

$$
t_{n+1}-t_{n}
$$

and the phase. According to (12) and (13), the jumps occur at time intervals (113) so that

$$
\int_{t_{n}+\epsilon}^{t_{n+1}+\epsilon} \dot{\phi}(\tau) \mathrm{d} \tau=2 \pi
$$

holds. Because of (97), (101) and (103), we obtain

$$
\dot{\phi}(\tau)=c+\mathrm{e}^{-\gamma\left(\tau-t_{n}\right)} x\left(t_{n}+\epsilon\right) .
$$


Inserting this relation into (114), we obtain

$$
\begin{aligned}
& c\left(t_{n+1}-t_{n}\right) \\
& \quad+x\left(t_{n}+\epsilon\right) \frac{1}{\gamma}\left(1-\mathrm{e}^{-\gamma\left(t_{n+1}-t_{n}\right)}\right)=2 \pi,
\end{aligned}
$$

which is an equation for (113) provided $x\left(t_{n}+\epsilon\right)$ is known. For a small damping constant of the dendritic currents, we expect

$$
\gamma\left(t_{n+1}-t_{n}\right) \ll 1 .
$$

Under this condition, (116) acquires the form

$$
\left(t_{n+1}-t_{n}\right)\left(c+x\left(t_{n}+\epsilon\right)\right)=2 \pi,
$$

or, because of (115), the form

$$
\left(t_{n+1}-t_{n}\right)=\frac{2 \pi}{\dot{\phi}\left(t_{n}+\epsilon\right)} .
$$

Equation (119) tells us that the sequence of jumptimes is inversely proportional to the speed of the phase, which is quite a reasonable result.

Let us now consider the steady state in which

$$
x\left(t_{n+1}+\epsilon\right)=x\left(t_{n}+\epsilon\right)
$$

holds. This implies that even in the general case (116) the jump-times are equidistant

$$
t_{n+1}-t_{n}=\Delta \text { equidistant. }
$$

This allows us to perform the sums that occur in (107) and (109) explicitly and the solution of Eq. (98) can be written as

$$
x\left(t_{N}+\epsilon\right)=\mathrm{e}^{-\gamma t_{N}} x_{1}\left(t_{0}\right)+A \frac{1-\mathrm{e}^{-\gamma N \Delta}}{1-\mathrm{e}^{-\gamma \Delta}},
$$

whereby we use the abbreviation (110). When we ignore transients, i.e. consider the steady state, (122) simplifies to

$$
x \equiv x\left(t_{N}+\epsilon\right)=A\left(1-\mathrm{e}^{-\gamma \Delta}\right)^{-1} .
$$

From (115) we then obtain

$$
\dot{\phi}\left(t_{n}+\epsilon\right)=c+A\left(1-\mathrm{e}^{-\gamma \Delta}\right)^{-1} .
$$

Because of the coupling $\sim A$, the phase velocity is increased. We can now determine $\Delta$ explicitly. We insert $x\left(t_{n}\right)$ according to (123) into (116) and obtain

$$
\Delta=\frac{1}{c}\left(2 \pi-\frac{A}{\gamma}\right)
$$

Clearly, the coupling strength $A$ must be sufficiently small, i.e. $A<2 \pi \gamma$.

So far we calculated the time-derivative of $\chi$. It is a simple matter to repeat all the steps done before so that we are able to derive the results for $\chi$ at time $T_{N}$ and also for $\phi$ at $T_{N}$. Under the assumption of equidistant jumps, we obtain

$$
\chi\left(t_{N}\right)-\chi\left(t_{0}\right)=\frac{1}{\gamma} \dot{\chi}\left(t_{0}\right)\left(1-\mathrm{e}^{-\gamma N \Delta}\right)
$$

and

$$
\phi\left(t_{N}\right)=\chi\left(t_{0}\right)+\frac{1}{\gamma} \dot{\chi}\left(t_{0}\right)\left(1-\mathrm{e}^{-\gamma N \Delta}\right) .
$$

Under the choice of the initial time, such that

$$
\chi\left(t_{0}\right)=\dot{\chi}\left(t_{0}\right)=0,
$$

we obtain in the limit of time $t \rightarrow \infty$, i.e. for the steady state,

$$
\phi\left(t_{N}\right)=\frac{1}{\gamma} A N+C N \Delta-\frac{A}{\gamma} \frac{1}{1-\mathrm{e}^{-\gamma \Delta}} .
$$

\section{FREQUENCY PULLING AND MUTUAL ACTIVATION OF TWO NEURONS}

We generalize Eq. (96) to those for two coupled neurons

$$
\begin{aligned}
& \ddot{\phi}_{1}+\gamma \dot{\phi}_{1}=A f\left(\phi_{2}\right)+C_{1}, \\
& \ddot{\phi}_{2}+\gamma \dot{\phi}_{2}=A f\left(\phi_{1}\right)+C_{2} .
\end{aligned}
$$

In analogy to (97) we make the substitution

$$
\phi_{j}=\frac{1}{\gamma} C_{j} t+\chi_{j}=c_{j} t+\chi_{j}, \quad j=1,2
$$


and obtain

$$
\begin{aligned}
& \ddot{\chi}_{1}+\gamma \dot{\chi}_{1}=A f\left(\chi_{2}+c_{2} t\right), \\
& \ddot{\chi}_{2}+\gamma \dot{\chi}_{2}=A f\left(\chi_{1}+c_{1} t\right) .
\end{aligned}
$$

Because of the cross-wise coupling in (130) and (131), the jump-times of $\dot{\chi}_{1}$ are given by $t_{n}^{(2)}$ and those of $\dot{\chi}_{2}$ by $t_{n}^{(1)}$. Otherwise we may proceed as in Section 4 and obtain for

$$
\begin{gathered}
t_{n}^{(2)}+\epsilon \leq t \leq t_{n+1}^{(2)}=\epsilon, \\
\dot{\chi}_{1}(t)=\dot{\chi}_{1}\left(t_{n}^{(2)}+\epsilon\right) \mathrm{e}^{-\gamma\left(t-t_{n}^{(2)}\right)},
\end{gathered}
$$

and, correspondingly for

$$
\begin{gathered}
t_{n}^{(1)}+\epsilon \leq t \leq t_{n+1}^{(1)}-\epsilon, \\
\dot{\chi}_{2}=\dot{\chi}_{2}\left(t_{n}^{(1)}+\epsilon\right) \mathrm{e}^{-\gamma\left(t-t_{n}^{(1)}\right)} .
\end{gathered}
$$

Furthermore we obtain the recursive equations (compare (102))

$$
\begin{aligned}
& \dot{\chi}_{1}\left(t_{n+1}^{(2)}+\epsilon\right) \\
& \quad=\dot{\chi}_{1}\left(t_{n}^{(2)}+\epsilon\right) \mathrm{e}^{-\gamma\left(t_{n+1}^{(2)}-t_{n}^{(2)}\right)}+A, \\
& \dot{\chi}_{2}\left(t_{n+1}^{(1)}+\epsilon\right) \\
& \quad=\dot{\chi}_{2}\left(t_{n}^{(1)}+\epsilon\right) \mathrm{e}^{-\gamma\left(t_{n+1}^{(1)}-t_{n}^{(1)}\right)}+A .
\end{aligned}
$$

Under steady-state conditions, where

$$
t_{n+1}^{(1)}-t_{n}^{(1)}=\Delta_{1}, \quad t_{n+1}^{(2)}-t_{n}^{(2)}=\Delta_{2}
$$

and

$$
\begin{aligned}
& \dot{\chi}_{1}\left(t_{n+1}^{(2)}+\epsilon\right)=\dot{\chi}_{1}\left(t_{n}^{(2)}+\epsilon\right) \equiv \dot{\chi}_{1}, \\
& \dot{\chi}_{2}\left(t_{n+1}^{(1)}+\epsilon\right)=\dot{\chi}_{2}\left(t_{n}^{(1)}+\epsilon\right) \equiv \dot{\chi}_{2},
\end{aligned}
$$

we obtain

$$
\begin{aligned}
& \chi_{1} \equiv \chi_{1}\left(t_{N}^{(2)}+\epsilon\right)=A\left(1-\mathrm{e}^{-\gamma \Delta_{2}}\right)^{-1}, \\
& \chi_{2} \equiv \chi_{2}\left(t_{N}^{(1)}+\epsilon\right)=A\left(1-\mathrm{e}^{-\gamma \Delta_{1}}\right)^{-1} .
\end{aligned}
$$

We now have to determine $\Delta_{1}$ and $\Delta_{2}$, which, in analogy to (114), are defined by

$$
\begin{aligned}
& \int_{t_{n}^{(1)}}^{t_{n+1}^{(1)}} \dot{\phi}_{1} \mathrm{~d} t=2 \pi, \\
& \int_{t_{n}^{(2)}}^{t_{n+1}^{(2)}} \dot{\phi}_{2} \mathrm{~d} t=2 \pi .
\end{aligned}
$$

When evaluating (146) and (147), we must observe that (136) and (138), and thus $\phi_{1}, \phi_{2}$ are defined only on intervals. To make our analysis as simple as possible (whereby we incidentally capture the most interesting case), we assume

$$
\left|\gamma \Delta_{1}\right| \ll 1, \quad\left|\gamma \Delta_{2}\right| \ll 1
$$

Then (146) and (147) read

$$
\begin{aligned}
& c_{1} \Delta_{1}+\dot{\chi}_{1} \Delta_{1}=2 \pi, \\
& c_{2} \Delta_{2}+\dot{\chi}_{2} \Delta_{2}=2 \pi,
\end{aligned}
$$

respectively, which because of (144), (145), and (148) can be transformed into

$$
\begin{aligned}
& c_{1} \Delta_{1}+\frac{A}{\gamma} \frac{\Delta_{1}}{\Delta_{2}}=2 \pi, \\
& c_{2} \Delta_{2}+\frac{A}{\gamma} \frac{\Delta_{2}}{\Delta_{1}}=2 \pi .
\end{aligned}
$$

Let us discuss these equations in two ways:

(1) We may prescribe $\Delta_{1}$ and $\Delta_{2}$ and determine those $c_{1}, c_{2}$ (that are essentially the neural inputs) that give rise to $\Delta_{1}, \Delta_{2}$.

(2) We prescribe $c_{1}$ and $c_{2}$ and determine $\Delta_{1}, \Delta_{2}$. Since $\omega_{j}=2 \pi / \Delta_{j}$ are the axonal pulse frequencies, we express our results by those

$$
\begin{aligned}
& \omega_{1}=2 \pi \frac{\left(c_{1} 2 \pi+c_{2} A / \gamma\right)}{4 \pi^{2}-A^{2} / \gamma^{2}}, \\
& \omega_{2}=2 \pi \frac{\left(c_{1} A / \gamma+c_{2} 2 \pi\right)}{4 \pi^{2}-A^{2} / \gamma^{2}} .
\end{aligned}
$$


Their difference and sum are particularly simple

$$
\begin{aligned}
\omega_{2}-\omega_{1} & =\frac{c_{2}-c_{1}}{1+A /(2 \pi \gamma)}, \\
\omega_{1}+\omega_{2} & =\frac{c_{1}+c_{2}}{1+A /(2 \pi \gamma)} .
\end{aligned}
$$

These results exhibit a number of remarkable features of the coupled neurons: according to (156) their frequency sum, i.e. their activity is enhanced by positive coupling $A$. Simultaneously, according to (155) some frequency pulling occurs. According to (153), neuron 1 becomes active even for vanishing or negative $c_{1}$ (provided $\left|c_{1} 2 \pi\right|<c_{2} A / \gamma$ ), if neuron 2 is activated by $c_{2}$. This has an important application to the interpretation of the perception of Kaniza figures, and more generally to associative memory, as we shall demonstrate elsewhere.

\section{MANY COUPLED NEURONS}

The case of two neurons can be generalized to many neurons. The corresponding equations read

$$
\begin{aligned}
\dot{\phi}+\gamma \phi_{j}= & \sum_{k} A_{j k}\left\{\phi_{k}-\phi_{k}(\bmod 2 \pi)\right\} \\
& +C_{j} t+B_{j}(t)
\end{aligned}
$$

where $j=1, \ldots, N$. Note that the coefficients $A_{j k}$ may be positive or negative according to excitatory or inhibitory couplings. In analogy to (33) we introduce a reference function $\phi$, i.e. the phase-locked state, by means of

$$
\dot{\phi}+\gamma \phi=A\{\phi-\phi(\bmod 2 \pi)\}+C t,
$$

where

$$
A=\sum_{k} A_{j k}
$$

is assumed to be independent of $j$. We put

$$
\phi_{j}=\phi+\xi_{j}
$$

and subtract (158) from (157) which yields

$$
\begin{aligned}
\dot{\xi}_{j}+\gamma \xi_{j}= & \sum_{k} A_{j k}\left\{\xi_{k}-\left(\phi+\xi_{k}\right)(\bmod 2 \pi)\right. \\
& +\phi(\bmod 2 \pi)\}+h_{j}(t)
\end{aligned}
$$

where

$$
h_{j}(t)=\left(C_{j}-C\right) t+B_{j}(t) .
$$

The formal solution of (161) reads

$$
\begin{aligned}
\xi_{j}^{(t)}= & \xi_{j}(0) \mathrm{e}^{-\gamma t}+\int_{0}^{t} \mathrm{e}^{-\gamma(t-\sigma)} h_{j}(\sigma) \mathrm{d} \sigma \\
& +\sum_{k} A_{j k} \kappa_{j}(t)
\end{aligned}
$$

where $\kappa_{j}(t)$ is the obvious generalization of $\kappa(t)$ in (36). Its evaluation for small $\left|\gamma \xi_{k}\right|$ yields, in analogy to the results (49), (55), and (56)

$$
\dot{\xi}_{j}+\gamma \xi_{j}=D(t) \sum_{k} a_{j k} \xi_{k}+h_{j}(t),
$$

where $a_{j k}=A_{j k} 2 \pi \dot{\phi}\left(t_{l}^{-1}\right)$ is independent of index $l$, because of stationarity of $\phi$, and

$$
D(t)=\sum_{\ell=-\infty}^{+\infty} \delta\left(t-t_{\ell}^{+}\right),
$$

where $t_{l}^{+}$is defined by $\phi\left(t_{l}\right)=2 \pi l, l$ integer.

The set of linear differential equations (164) can be solved by the standard procedure. We introduce eigenvectors $\tilde{v}^{\kappa}$ with components $v_{j}^{\kappa}$ so that

$$
\sum_{j} v_{j}^{\kappa} a_{j k}=\lambda_{\kappa} v_{k}^{\kappa}
$$

and put

$$
\begin{aligned}
& \sum_{j} v_{j}^{\kappa} \xi_{j}=\eta_{\kappa}, \\
& \sum_{j} v_{j}^{\kappa} h_{j}=\tilde{h}_{\kappa} .
\end{aligned}
$$

This allows us to transform (164) into the uncoupled equations

$$
\dot{\eta}_{\kappa}+\gamma \eta_{\kappa}=D(t) \lambda_{\kappa} \eta_{\kappa}+\tilde{h}_{\kappa} .
$$

Their solution can be obtained as in Section 3 . 


\section{MANY NEURONS WITH DIFFERENT INPUT STRENGTHS}

Generalizing the notation of the previous section, whereby we distinguish the different neurons by an index $j$, we first put

$$
x_{j}=\dot{\chi}_{j}, \quad \dot{\phi}_{\ell}=\dot{\chi}_{\ell}+c_{\ell}=x_{\ell}+c_{\ell} .
$$

The equations for $x_{j}$ then read

$$
\dot{x}_{j}+\gamma x_{j}=\sum_{\ell} A_{j \ell} f\left(\phi_{\ell}\right) .
$$

Since (171) is, at least from a formal point of view, a linear equation in $x_{j}$, we make the hypothesis

$$
x_{j}=\sum_{\ell} x_{j}^{(\ell)}
$$

and require

$$
\dot{x}_{j}^{(\ell)}+\gamma x_{j}^{(\ell)}=A_{j \ell} f\left(\phi_{\ell}\right) .
$$

Under the assumption of equidistant jumps and steady state, we may exploit the results of Sections 4 and 5 and obtain as solution of (173) the relation

$$
x_{j}^{(\ell)}\left(t_{N(\ell)}+\epsilon\right)=A_{j \ell}\left(1-\mathrm{e}^{-\gamma \Delta_{\ell}}\right)^{-1},
$$

or for an arbitrary time with $t_{N(l)}+\epsilon<T<$ $t_{N(l)+1}-\epsilon$

$$
x_{j}^{(\ell)}(T)=\mathrm{e}^{-\gamma\left(T-t_{N(\ell)}\right)} A_{j \ell}\left(1-\mathrm{e}^{-\gamma \Delta_{\ell}}\right)^{-1} .
$$

Using (172), we obtain the final result

$$
x_{j}(T)=\sum_{\ell} \mathrm{e}^{-\gamma\left(T-t_{N(\ell)}\right)} A_{j \ell}\left(1-\mathrm{e}^{-\gamma \Delta_{\ell}}\right)^{-1} .
$$

The jump-intervals are determined by

$$
\int_{t_{n}^{(\ell)}}^{t_{n+1}^{(\ell)}} \dot{\phi}_{\ell}(\sigma) \mathrm{d} \sigma=2 \pi .
$$

In order to evaluate the integral in (177), we use (170) and (176), where under the assumption

$$
\gamma\left(t_{N(\ell)+1}-t_{N(\ell)}\right) \ll 1,
$$

(176) can be approximated by

$$
x_{j}=\sum_{\ell^{\prime}} A_{j \ell^{\prime}} /\left(\gamma \Delta_{\ell^{\prime}}\right) .
$$

Thus we obtain (generalizing (151) and (152))

$$
c_{\ell} \Delta_{\ell}+\Delta_{\ell} \sum_{\ell^{\prime}} A_{\ell \ell^{\prime}} /\left(\gamma \Delta_{\ell^{\prime}}\right)=2 \pi .
$$

These equations relate the axonal pulse frequencies $\omega_{l}=2 \pi / \Delta_{l}$ to the strengths of the sensory inputs, $c_{l}$. The corresponding equations for $\omega_{l}$ are linear and read

$$
c_{\ell}+\sum_{\ell^{\prime}} A_{\ell \ell^{\prime}} /(2 \pi \gamma) \omega_{\ell^{\prime}}=\omega_{\ell}
$$

They can be solved under the usual conditions. Depending on the coupling coefficients $A_{l l^{\prime}}$, even those $\omega_{l}$ may become nonzero, for which $c_{l}=0$. On the other hand, only those solutions are allowed for which $\omega_{l}>0$ for all $l$. This imposes limitations on $c_{l}$ and $A_{l l^{\prime}}$.

\section{CONCLUDING REMARKS AND OUTLOOK}

In the above paper I treated a model that is highly nonlinear because of the dependence of the $\delta$ functions on the phases $\phi$. Nevertheless, at least in the limit of small dendritic damping, I could solve it explicitly. This model contains two thresholds. The first threshold is the conventional one where one assumes that below it the neuron is quiescent, whereas above threshold the neuron fires. It was assumed that the network operates below its second threshold, where we expect pronounced saturation effects on the firing rates. Probably this region has to be explored in more detail. Also the case that the dendritic damping is not small might deserve a further study. Preliminary considerations show that here chaotic firing rates must be expected. 


\section{References}

[1] H. Haken: What can synergetics contribute to the understanding of brain functioning? In: Analysis of Neurophysiological Brain Functioning, C. Uhl (Ed.), Springer, Berlin (1999)

[2] W. McCulloch and W. Pitts: A logical calculus of the ideas immanent in nervous activity, Bull. Math. Biophys. 5, $115-133$ (1943).

[3] H. Braun: Stochastic encoding with intrinsically oscillating neurons, Talk given at Sigtuna workshop (1998).
[4] R.E. Mirollo and S.H. Strogatz: SIAM (Soc. Ind. Appl. Math.) J. Appl. Math. 50, 1645 (1990).

[5] U. Ernst, K. Pawelzik and T. Geisel: Phys. Rev. E, 57(2) (1998), with many further references.

[6] Y. Kuramoto and I. Nishikawa: J. Stat. Phys. 49, 569 (1987)

[7] Y. Kuramoto: Physica D50, 15 (1991).

[8] P. Tass and H. Haken: Z. Phys. B 100, 303-320 (1996)

[9] P. Tass and H. Haken: Biol. Cybern. 74, 31-39 (1996). 


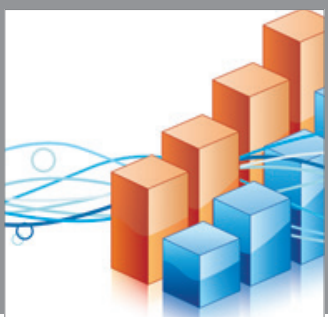

Advances in

Operations Research

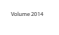

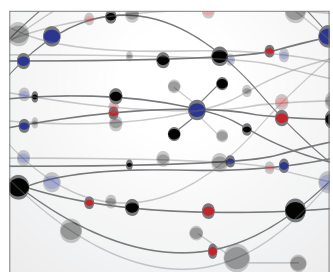

\section{The Scientific} World Journal
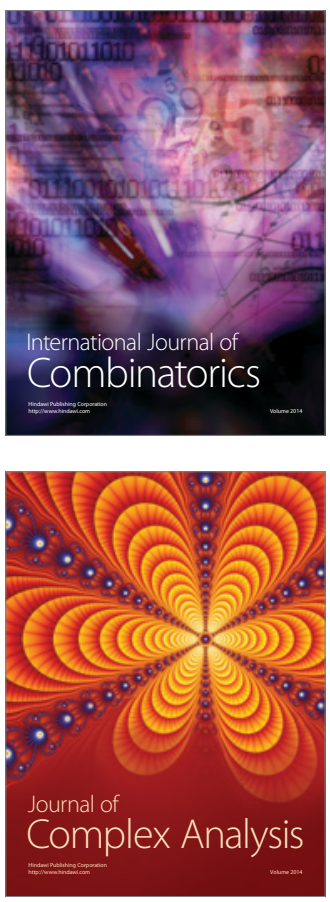

International Journal of

Mathematics and

Mathematical

Sciences
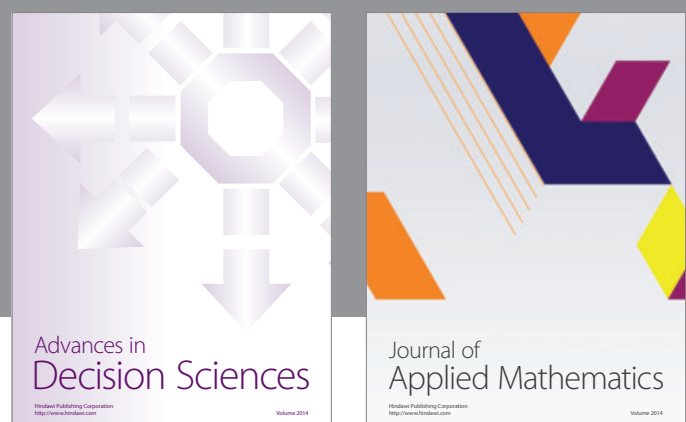

Journal of

Applied Mathematics
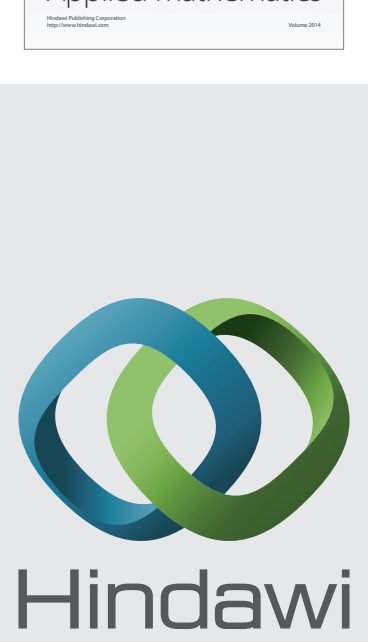

Submit your manuscripts at http://www.hindawi.com
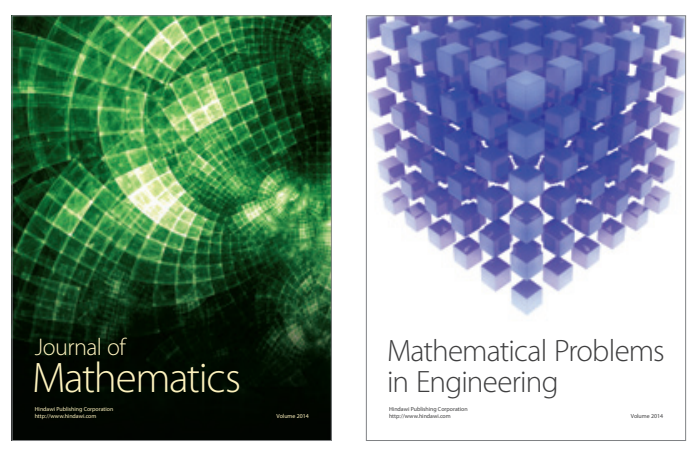

Mathematical Problems in Engineering
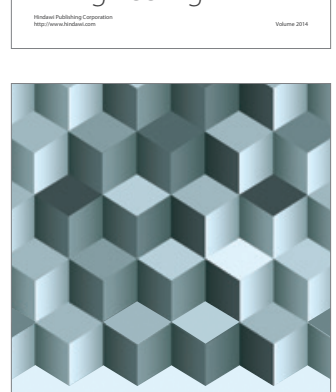

Journal of

Function Spaces
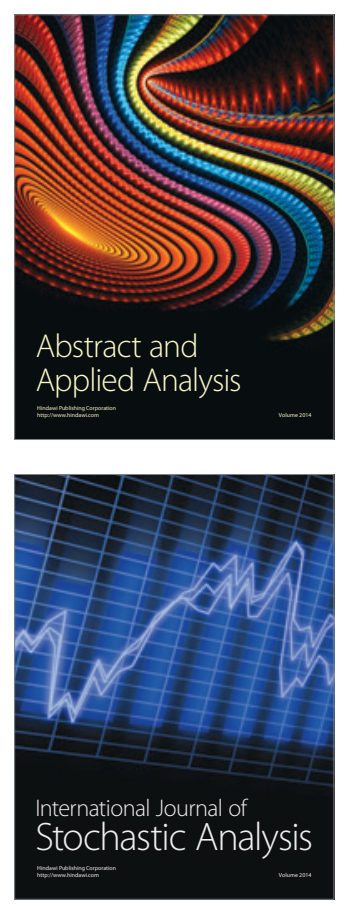

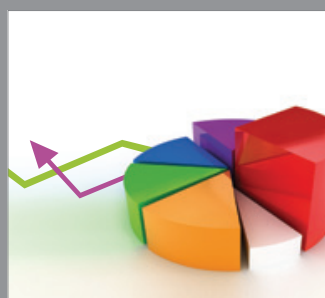

ournal of

Probability and Statistics

Promensencen
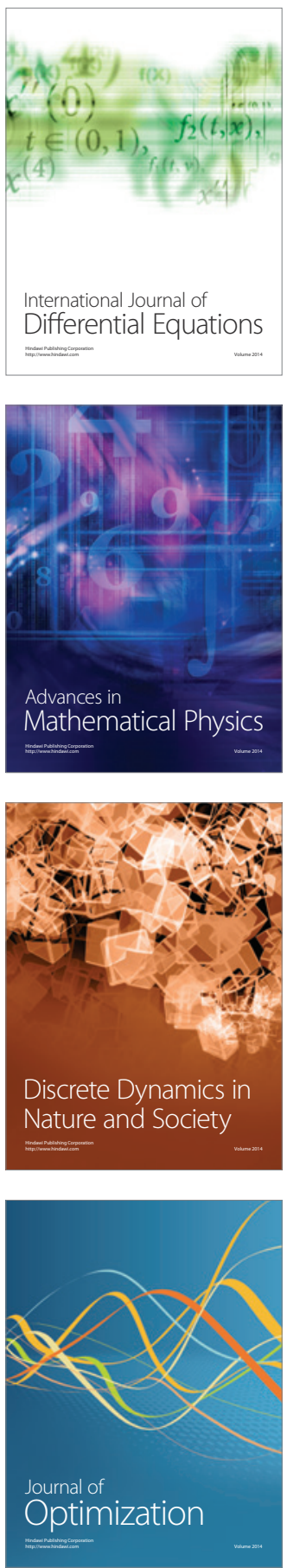